\title{
Problema de Programação da Produção um Esquema de Classificação
}

\author{
Oscar Ciro López, Dr. ${ }^{1}$ \\ Prof.Ricardo M. Barcia, Ph.D ${ }^{1}$ \\ Prof. Osama Eyada, Ph.D 2
}

${ }^{1}$ Departamento de Engenharia de Produção e Sistemas

Programa de Pós-Graduação em Engenharia de Produção

Cx.P. 476 Tel: (048) 2341255 / 2319596

Universidade Federal de Santa Catarina, CEP 88040-900 - Florianópolis - SC

${ }^{2}$ Department of Industrial and System Engineering

Virginia Polytechnic Institute and State University

Blacksburg, Virginia 24061-0118 - USA

Palavras Chave: Programação, Sequenciamento, Problemas de Produção, Classificação da Produção

Key Words: Scheduling, Sequencing, Production Problems, Production Classification

\section{RESUMO}

Muitas vezes, não é simples encontrar uma classificação exata para os problemas de programação, não somente porque existem diferentes versões para um dado problema, mas, porque vários procedimentos para uma questão particular, são caracterizados por premissas diferentes e limitações de aplicação dos modelos desenvolvidos. O objetivo deste artigo é delinear uma classificação ampla que permita estabelecer o sentido, direção e perspectiva de pesquisas conduzidas na área. $O$ trabalho não tem a intenção de dar um levantamento exaustivo da literatura de programação da produção, que pode ser encontrado em vários outros trabalhos de revisão.

\section{ABSTRACT}

It is the purpose of this article to review the various solutions that have been proposed for the production scheduling problem. An attempt is made to give a classification scheme to categorize the existing procedures that allow to point out potential future courses of development. Emphasis is placed on the basic assumptions involved in each production sequencing problem rather than to approaches used to obtain a solution. 


\section{Introdução}

O problema de programação pode ser definido, de um modo geral, como a alocação de recursos no tempo de forma a executar um conjunto de tarefas (MACCARTHY \& LIU, 1993). Este conceito é de vital importância para várias atividades industriais, particularmente nos ambientes de manufatura e de projetos de construção.

Tipicamente, o problema de programação da produção envolve um conjunto de produtos que devem ser completados, onde cada produto utiliza um conjunto de operações para ser executado. As operações necessitam de máquinas e recursos materiais e devem ser realizadas seguindo alguma seqüência tecnológica. Desenvolver uma programação da produção envolve a seleção de uma seqüência de operações (ou rotas de processo) que resultará na realização do produto. Envolve também, a determinação dos recursos necessários para executar cada operação da rota, bem como a determinação das datas em que cada operação da rota começará e terminará (RODAMMER \& WHITE, 1988). Portanto, a programação da produção é um problema que decide a ordem de execução de todos os produtos em cada máquina e que determina a data de início de cada operação de forma a otimizar uma função objetivo (BELLMAN et al. 1982).

Os problemas de programação da produção são reconhecidamente caracterizados por uma inerente dificuldade de solução, muitos dos quais são
NP-pesados, indicativo da intratabilidade de grandes e, frequientemente, de problemas de tamanho moderados. Neste casos, é provável que seja necessário o emprego de heurísticas para encontrar uma aproximação da solução ótima.

Muitas vezes, não é simples encontrar uma classificação exata para os problemas de programação, não somente porque existem diferentes versões para um dado problema, mas, porque vários procedimentos para uma questão particular, são caracterizados por premissas diferentes e limitações de aplicação dos modelos desenvolvidos.

A intenção deste trabalho não é dar um levantamento exaustivo da literatura de programação da produção, que pode ser encontrado em vários trabalhos de revisão, tais como, Elmaghraby (1968), Bakshi e Arora (1969), Day e Hottenstein (1970), Lenstra et al. (1977), Panwalker e Iskander (1977), Godin (1978), Graham et al. (1979). King e Spachis (1980), Graves (1981), Rodammer e White Jr. (1988), Buxey (1989), Hendry e Kingsman (1989), Baker e Scudder (1990) e MacCarthy B.L. e Liu J. (1993). O objetivo aqui é delinear uma classificação ampla que permita estabelecer o sentido, direção e perspectiva de pesquisas conduzidas na área, assim como oferecer um resumo das principais abordagens e paradigmas desenvolvidos.

\section{Problema de Programação da Produção}

O problema geral de programação da produção pode ser definida como: um 
conjunto de $n$ produtos $\left\{J_{1}, J_{2}, \ldots, J_{n}\right\}$ deve ser processado em $m$ máquinas $\left\{M_{1}, M_{2}, \ldots\right.$, $M_{m}$ \} disponíveis. Um subconjunto destas máquinas é requerido para completar o processamento de cada produto. $O$ padrão de fluxo nas máquinas pode ou não ser fixado para algum ou todos os produtos. $\mathrm{O}$ processamento do produto $J_{i}$ na máquina $M_{i}$ é denominado de uma operação, denotado por $O_{i j}$. Para cada operação $O_{i j}$, existe um tempo de processamento $t_{\mathrm{ij}}$. associado $\mathrm{O}$ problema consiste em encontrar uma programação que otimize alguma medida de desempenho (MACCARTHY \& LIU, 1993)..

Está bem estabelecido que a maioria do problemas de programação da produção pertencem a classe dos NP-completos. Trabalhos relativos à complexidade $\mathrm{e}$ provas da NP-dureza de novos e velhos e já sedimentados modelos de programação da produção podem ser encontrados em Ullman (1975), Rinnooy Kan (1976); Ullman (1976), Garey et al. (1976), Lenstra et al. (1977), Lenstra (1977), Ibaraki (1977), Lenstra e Rinnooy Kan (1978), King e Spachi (1980), Goyal e Sriskandarajah (1988), Monma e Potts (1989), Kamoun e Sriskandarajah (1993), Chen e Bulfin (1993).

\section{Abordagens para o Problema}

A grande maioria de procedimentos de programação, podem ser categorizados em dois principais grupos, baseados nas abordagens empregadas. o primeiro, e de longe a maior categoria, denominado de procedimentos heurísticos objetiva produzir programações viáveis boas. $\mathrm{O}$ segundo grupo consiste dos procedimentos que procuram produzir a programação melhor ou ótima. Estes são denominados de procedimentos ótimos, também chamados exatos ou analíticos porque geralmente envolvem alguma forma de programação matemática ou outro procedimento analítico mais rigoroso.

Dentro de cada um destes grupos há possíveis esquemas de sub-categorização. Os procedimentos heurísticos por exemplo, podem ser divididos em duas classes (STORER, WU \& VACCARI, 1992):

1.heurísticas específicas, desenvolvidas para cada tipo de problema, baseadas nas especificações da questão tratada (ex. ${ }^{1}$ regras de dispatching e sequenciamento e abordagens de Sistemas Especialistas) e,

2.heurísticas de busca local, que são formas alternativas de busca do espaço de soluções baseadas no princípio da vizinhança ${ }^{2}$,como por exemplo: hill climbing, steepest descent, Simulated Annealing ( $S A)$, Algoritmos Genéticos (AG) e Tabu Search.

Em relação aos procedimentos ótimos, outras duas classes principais podem ser identificadas (MACCARTHY \& LIU, 1993):

1.métodos ótimos eficientes: são métodos que geram programações ótimas em tempo polinomial, e

2.métodos ótimos enumerativos: são 
métodos que envolvem uma enumeração parcial do conjunto de todas as programações possíveis. Esta abordagem é desenvolvida baseada em dois conceitos principais (DAY \& HOTTENSTEIN, 1970): a) o uso de uma técnica de enumeração controlada para considerar todas as soluções potenciais e, b) a eliminação de potenciais soluções inaceitáveis. As mais gerais são formulações de programação matemática, métodos de branch and bound e métodos de eliminação.

\section{Classificação dos Problemas de Programação da Produção}

Diversos esquemas tem sido propostos para a categorização dos problemas de programação da produção. Conway et al. 1967 (in: MACCARTHY B.L. \& LIU J. 1993) sugere um esquema de classificação baseado em quatro descritores $A / B / C / D$, onde $A$ representa o número de produtos, $B$ representa o número de máquinas, $C$ representa o padrão de fluxo e futuras restrições tecnológicas e de administração e, $D$ representa o critério a ser otimizado Por exemplo, $n / m / J / C m a x$ refere-se ao problema job shop com $n$ produtos e $m$ máquinas que objetiva minimizar o tempo total de processamento (makespan).

Lenstra (1977) introduz um parâmetro $\lambda$ ao terceiro elemento desse esquema tradicional, para representar algumas variações relevantes. A notação $n / m / 1, \lambda l k$, é utilizada para representar nos dois primeiros parâmetros o número de produtos e de máquinas respectivamente, $I$ assume um valor de acordo com o padrão de fluxo e o quarto elemento indica o critério de otimalidade.

Lenstra e Rinnooy Kan (1978) caracteriza cada problema de programação em outra classificação de quatro parâmetros $\alpha / \beta / \gamma / \delta$, onde $\alpha$ indica o número de máquinas, $\beta$ indica ou o tipo de restrições de precedências (prec ou tree) ou a ausência delas, $\gamma$ indica ou a restrição que o tempo de processamento $p_{i}$ pertence à $\{\gamma\}$ para todo $i$ pertencente à $I$, ou a ausência da mesma, $\delta$ representa o critério de otimalidade.

Graham et al. (1979) apresenta uma notação baseada em três parâmetros $\alpha / \beta / \gamma$ onde o.primeiro elemento define o número de máquinas e o padrão de fluxo, $\beta$ indica alguma restrição sobre os produtos e $\gamma$ define o critério de programação.

O esquema aqui proposto para a classificação dos problemas de programação é baseado nas características gerais dos problemas e as hipóteses usuais feitas sobre os produtos, máquinas e tempos de processamento. Esta categorização pode ser considerada como uma macro-divisão dos problemas de programação da produção.

De acordo com King e Spachis (1980), Bellman et al. (1982) e Forst (1984) as premissas básicas para os produtos, máquinas e tempos de processamento geralmente assumidas na literatura são: 


\section{Premissas para os produtos}

- Todos os $n$ produtos a serem realizados estão simultaneamente disponíveis na data zero.

- As operações não podem ser interrompidas. Qualquer operação, uma vez começada deve continuar até a sua completa realização.

- Cada produto consiste de $m$ operações que devem ser processadas em $m$ máquinas.

- O processamento de qualquer operação não pode ser feito por mais do que uma máquina por vez.

- Não há ordem de prioridade entre os produtos e cada um tem o mesmo grau de importância

- E permitido estoques ilimitados de produtos em processo ou tempos de espera intermediários

- Uma data final (due-date) pode ser atribuída a cada produto.

- Cada produto pode ser processado em cada máquina uma única vez

\section{Premissas para as máquinas}

- A capacidade das máquinas e da mão-de-obra é fixa

- Todas as $m$ máquinas estão disponíveis no início. Quebras e reparações não são permitidas durante o processo.

- Qualquer máquina pode processar qualquer produto.

- Existe uma máquina de cada tipo ou são arranjadas para trabalhar em paralelo uma independentemente da outra.

- Uma máquina não pode executar mais do que uma operação por vez

- Não é possível o intercâmbio de máquinas (flexibilidade).
3. Premissas para os tempos de processamento

- O tempo de processamento de cada operação é dado no início e permanece constante, independentemente da ordem de execução.

- O tempo de preparação e de transporte é incluído no tempo de processamento de uma operação.

\section{Esquema de Classificação Proposto}

Vários problemas de programação da produção são caracterizados relaxando ou restringindo algumas das premissas descritas na seção anterior. Uma grande gama de resultados e variações existem para um vasto conjunto de especificações ou restrições dos problemas.

Por exemplo, pode-se encontrar problemas com: restrições de precedências comuns entre produtos $\left(J_{i}\right.$ precede $J_{j}$ ), restrições de precedências entre produtos do tipo árvore (o grafo de precedência pode ter a forma de ramificações), possíveis datas de liberação não iguais para os produtos, não tem datas de espera (no wait) para os produtos entre o seu início e o tempo de realização, um limite superior constante para o número de operações por produtos, um limite superior constante para o tempo de processamento, rotas de processo estáticas, onde cada produto é executado em cada máquina exatamente um vez, $\mathrm{cm}$ estrita seqüência tecnológica (entre produtos) ou, rotas de processamento 
dinâmicas, onde a rota de processamento pode mudar dinamicamente pelo estado da planta (ex. disponibilidade de recursos) ou pelas condições do trabalho (ex. necessidade de retrabalho).

Consequentemente, com isto em mente, um esquema para classificar os problemas de produção é ilustrado na Figura 1.

Este esquema mostra que os problemas de programação podem ser classificados de acordo com:

1. As restrições tecnológicas dos produtos (COFFMAN JR. 1976)

- O caso de processamento sem interrupção: cada operação uma vez iniciada deve ser executada até o seu término;

- O caso com interrupção: uma tarefa pode ser interrompida e removida do processador sob a hipótese de que, eventualmente, todo o tempo de processamento será realizado, e que não há perda do tempo já empregado devido à interrupção, ou seja, as tarefas interrompidas reiniciam a suas execuções no ponto que foram interrompidas.

2. O critério de programação (GRAVES 1970, RODAMMER \& WHITE 1988 E MACCARTHY \& LIU, 1993)

O critério indica a medida sobre a qual as programações, sob qualquer conjunto de premissas, são avaliadas. Neste sentido, duas classes de problemas podem ser identificadas:

- Problemas de único critério: as formulações tradicionais de programação da produção, geralmente especificam um único critério, usualmente associados com custos ou desempenhos das programações. Critérios associados aos custos incluem custos de permanência de estoques, produção variável e custo de atrasos etc. Medidas comuns do desempenho da programação estão relacionados çom a utilização eficiente dos níveis dos recursos de produção (makespan, $C \max$ ), rápida resposta à demanda (tempo médio de processamento $C$, tempo médio de fluxo $F$, tempo médio de espera $W$ e cumprimento de datas limites (atraso médio $T$, atraso máximo $T_{\max }$, número de produtos atrasados $N_{T}$ ).

- Múltiplos critérios: ambientes atuais de produção abrangem múltiplas, conflitantes e algumas vezes não comensuráveis restrições e objetivos de desempenho, como minimizar stresses operacionais, melhorar a estabilidade da programação, redução da ociosidade de máquinas para diminuir filas e estoques ou para assegurar a confiabilidade.

\section{A geração de solicitações (GRAVES} 1970)

- open shop: todas as ordens de produção são solicitações dos clientes e não há estoques finais

- closed shop: todas as solicitações dos clientes são atendidas pelos estoques e as tarefas de produção são geralmente, resultados de decisões de reposição de estoques. Este problema é mais conhecido como problema de tamanho de lote.

Outros nomes empregados para esta divisão são: sistemas Make-to-Order e Make-to-Stock respectivamente (HENDRY \& KINGSMAN, 1989). 


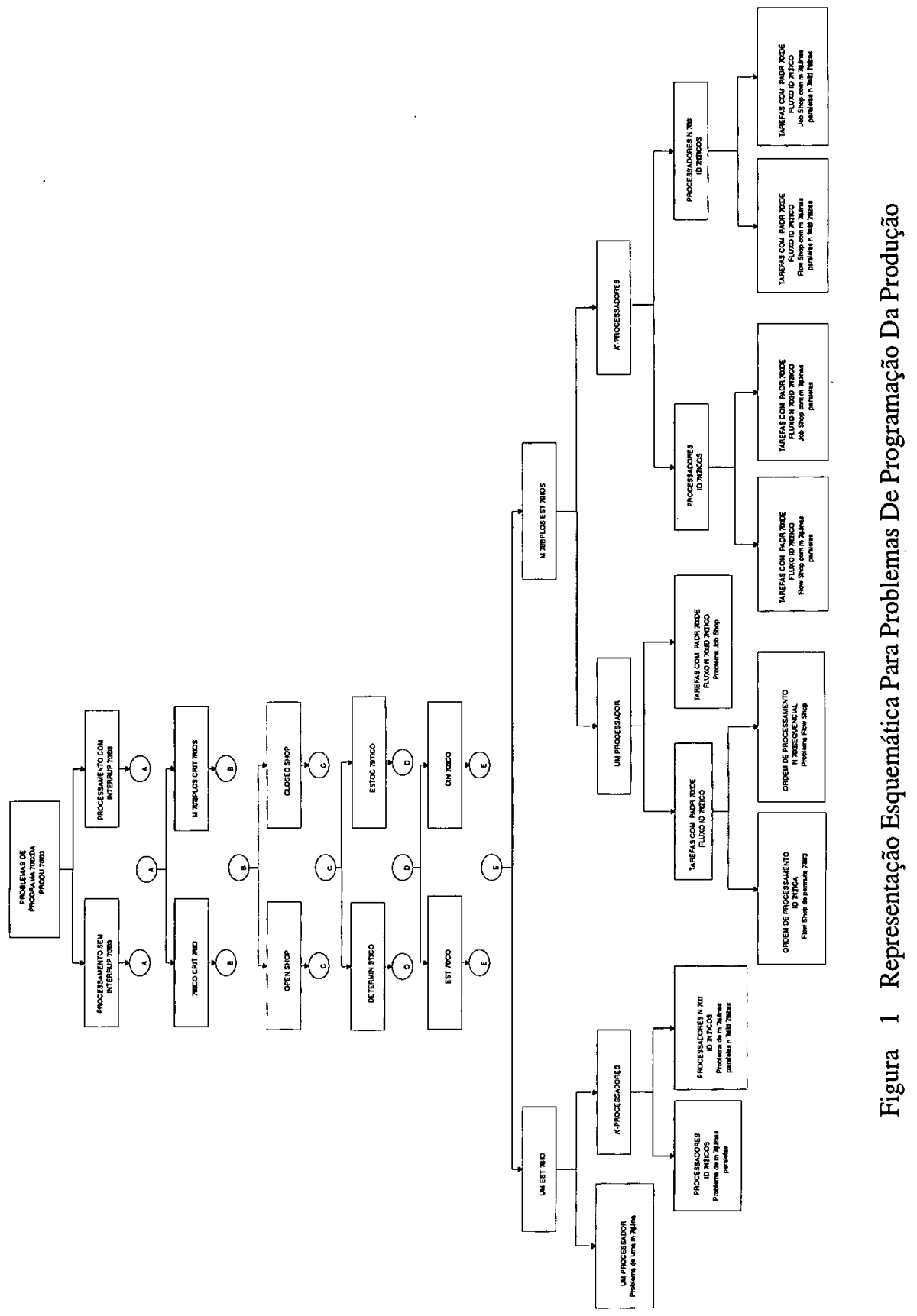


4. O ambiente de programação (RODAMMER \& WHITE 1988; GRAVES 1970; BELLMAN et al. 1982)

- Determinístico: o problema de programação é definido com respeito a um conjunto finito de solicitações totalmente especificadas, isto é, tempos de processamentos, tempos de liberação e datas de entrega são determinísticos. Produtos, máquinas, mão-de-obra e recursos materiais estão disponíveis para todos ao longo do processo.

- Estocásticos: o problema estocástico é definido levando em consideração eventos e distúrbios aleatórios, tais como: quebras de máquinas, indisponibilidade de operador e de material, tempos variáveis de processamento, de liberação e de entrega.

5. A natureza da chegada do produto (DAY \& HOTTENSTEIN 1970)

- Caso estático: todos os produtos são simultaneamente iniciados.

- Caso dinâmico: os produtos estão constantemente entrando e saindo da fila, de acordo com algum processo estocástico

6. A complexidade de processamento (GRAVES 1970, MACCARTHY \& LIU 1993)

A complexidade de processamento está relacionada com o número de passo associados com cada tarefa de produção

- Um estágio com um processador: todas as tarefas requerem um passo de processamento que deve ser executado em um elemento de produção. este problema é comumente denominado de problema de uma máquina.

- Um estágio com processadores paralelos: as tarefas podem ser executadias em $k$ processadores idênticos ${ }^{3}$ ou não idênticos num único estágio. Cada produto necessita uma, e somente uma destas máquinas

- Múltiplos estágios, flow shop: para um problema de múltiplos estágios, cada tarefa requer ser processada num conjunto de máquinas distintas. Nos problemas flow shop, cada produto tem um padrão de fluxo idêntico. Um problema flow shop no qual a ordem de processamento dos produtos em todas as máquinas é restrito de forma a ser a mesma é denominado de flow shop de permutação.

- Múltiplos estágios, job shop: aqui cada produto tem o seu próprio padrão de fluxo ou rota específica através das máquinas. Isto quer dizer que não há restrição no passos de processamento para as tarefas, e que rotas alternativas para os produtos são permitidas.

- Múltiplos estágios, flow shop com máquinas paralelas: é um problema flow shop onde há $k_{i}$ máquinas idênticas ou não idênticas, em um ou em todos os estágios $(i=1,2, \ldots, m), \mathrm{e}$ qualquer produto requerendo este ẹstágio deve ser processado em uma, e somente uma, destas máquinas.

- Múltiplos estágios, job shop com máquinas paralelas: é um problema job shop onde há $k_{i}$ máquinas idênticas ou não idênticas, em um ou em todos os estágios $(i=1,2, \ldots, m)$, e qualquer produto requerendo este estágio deve ser processado em uma, e somente uma, destas máquinas. A situação mais geral é o problema job shop em máquinas paralelas não idênticas. 
Como resumo do esquema proposto para a categorização dos problemas de programação da produção, alguns trabalhos são listados nas figuras 2 à 5 . Deve ser observado que a presença de restrições adicionais foramomitidas dada a grande variedade de problemas. As referências citadas tem a intenção de ser exemplos e não são necessariamente exaustivas.

\begin{tabular}{|c|c|}
\hline & Princlpala Objetlvos Uedoe Para Medir O Dewernpento Ds Progremeçlo \\
\hline$c_{1}$ & Tempo de processemento \\
\hline $\bar{C}$ & Tompo modio de processamento \\
\hline$s w_{i} C_{i}$ & Some ponderede do tempo de processamento \\
\hline$c_{\max }$ & Tempo maxtmo de processamento (makespan) \\
\hline$F_{j}$ & Tompo de nuxo \\
\hline$s w_{l} F_{j}$ & Soma ponderada do tempo de fixoo \\
\hline $\bar{F}$ & Tempo de tuxo médio \\
\hline$s w_{i} \bar{F}$ & Soma ponderado do tempo mbdio de fluxo \\
\hline ws & Tompo do ospera \\
\hline$\vec{W}$ & Tempo modro de espers \\
\hline$s w, w$ & Some pondereda do tempo de espere \\
\hline$T_{i}$ & Tandoza \\
\hline $\bar{T}$ & Tondeze madia \\
\hline$T_{\operatorname{mox}}$ & Tandeza máxima \\
\hline$S w_{j} T_{j}$ & Some penderada de tardezs \\
\hline 4 & Atraso \\
\hline $\bar{L}$ & Atraso metio \\
\hline $\operatorname{Lim}_{\operatorname{mix}}$ & Atraso máxtmo \\
\hline$S w_{j} L_{j}$ & Some ponderada de atraso \\
\hline $\mathrm{Nr}_{\mathbf{r}}$ & Namero de produtos atrasados \\
\hline$S w_{j} N_{r}$ & Soma ponderada de proditos atrasados \\
\hline$E_{1} / T_{1}$ & Cado e Tarde \\
\hline st & Tempo de inlcio \\
\hline$S w_{j} E_{j} / w_{i} T_{j}$ & Some pondereda de cedos e tardes \\
\hline VAR & Vasiancla do tempo de processamento \\
\hline COVAR & Coeficiente de variagso \\
\hline BICRET & Terrpo mécio de processamento e variancia do tempo de processamento \\
\hline FV & Variancla do tempo de fluxo \\
\hline crv & Varlancta mida do termpo de processamento \\
\hline NPV & Vator presente da nede \\
\hline MWAD & Desvio absoluto ponderado do tempo de processamento de date de realzegăto \\
\hline$f_{\max }$ & Custo maxtmo (ou tolal) \\
\hline$\rho_{\max }$ & Luero máxhmot \\
\hline
\end{tabular}




\begin{tabular}{|c|c|c|c|}
\hline Funģăo Objetivo & Método & Refertncia & Observaçbes \\
\hline & $\begin{array}{l}\text { otimo } \\
\text { otimo } \\
\text { ótimo } \\
\text { otimo e heurística }\end{array}$ & $\begin{array}{l}\text { Smith, 1956, (veja } \\
\text { MeCarthey e Liu 1993) } \\
\text { Glazabrook, } 1976 \text { (veja } \\
\text { Forst, 1984) } \\
\text { Szwarc et al. } 1988 \\
\text { Chandiv et al. } 1993\end{array}$ & $\begin{array}{l}\text { Tempo de processamento } \\
\text { estocástico } \\
\text { Batch processing } \\
\text { machine }\end{array}$ \\
\hline$w_{j} C_{j}$ & ótimo & $\begin{array}{l}\text { Held e Karp, } 1962 \text { (veja } \\
\text { MacCarthey e Liu, 1993) }\end{array}$ & \\
\hline$w_{j} C_{j} \in F_{i}$ & ótimo & Cheng, 1891 & \\
\hline$C$ & & \begin{tabular}{ll|}
$\begin{array}{l}\text { Rothkopf, } \\
\text { Forst, 1984) }\end{array}$ & (veja \\
\end{tabular} & $\begin{array}{l}\text { Tempo de processamento } \\
\text { estocástico }\end{array}$ \\
\hline$C_{\max }$ & $\begin{array}{l}\text { otimo } \\
\text { hourlstica } \\
\text { otimo } \\
\text { otimo }\end{array}$ & $\begin{array}{l}\text { Dauzere-Peres, } 1993 \\
\text { Alidaee, } 1990 \\
\text { Glazebrook, } 1992 \\
\text { Chu, } 1992\end{array}$ & $\begin{array}{l}\text { Tempo de processamento } \\
\text { estocástico, interrupcáo e } \\
\text { náo interrupcăo } \\
\text { datas de liberacăo } \\
\text { desiguais }\end{array}$ \\
\hline$C_{\max } \theta N_{T}$ & otimo & Kiran e Unal, 1991 & Múltiplos critérios \\
\hline 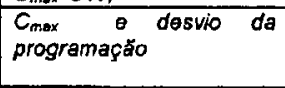 & heuristica & Wu et al. 1993 & $\begin{array}{l}\text { Múltiplos critérios. } \\
\text { Permite descontinuidade } \\
\text { na máquina. }\end{array}$ \\
\hline$\Sigma w_{j} C_{j}$ & $\begin{array}{l}\text { ótimo } \\
\text { ótimo } \\
\text { ótimo } \\
\text { heuristica } \\
\text { heuristica }\end{array}$ & $\begin{array}{l}\text { Smith, 1956, (veja } \\
\text { MacCarthey e Liu, 1993) } \\
\text { Horn, 1972 (veja King e } \\
\text { Spachis, 1980) } \\
\text { Sidney, 1975: } 1977 \text { (veja } \\
\text { King e Spachis. 1980) } \\
\text { Morton \& Dharan, 1978 } \\
\text { (veja MacCarthey e Liu, } \\
\text { 1993) } \\
\text { Weiss, 1981 (veja } \\
\text { MacCarthy e Liu, 1993) }\end{array}$ & 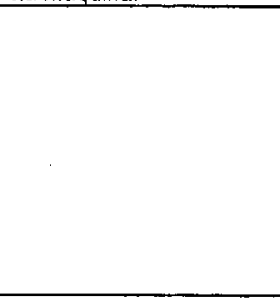 \\
\hline CTV & $\begin{array}{l}\text { heurlstica - genetic } \\
\text { algorithms } \\
\text { otimo }\end{array}$ & $\begin{array}{l}\text { Gupta et al. } 1993 \\
\text { De et al. } 1992\end{array}$ & \\
\hline$L_{\max }$ of $T_{\max }$ & otimo & $\begin{array}{l}\text { Jackson, } 1955 \text { (veja } \\
\text { MacCarhy e Liu, 1993) }\end{array}$ & \\
\hline$L_{\max }$ & $\begin{array}{l}\text { otimo } \\
\text { heuristica } \\
\text { otimo }\end{array}$ & $\begin{array}{l}\text { Lawler, } 1973 \text { (veja Klng e } \\
\text { Spachis, 1980) } \\
\text { Lee, } 1991 \\
\text { Leon e Wu, } 1992\end{array}$ & \\
\hline$L_{\max }, T_{\max }$ & & $\begin{array}{l}\text { Crabill e Maxwell, } 1969 \\
\text { (veja Forst, 1984) }\end{array}$ & $\begin{array}{l}\text { Tempo de processamento } \\
\text { estocático e "due dates". }\end{array}$ \\
\hline$T_{\text {max }}$ & $\begin{array}{l}\text { ótimo } \\
\text { ótimo } \\
\text { ótimo } \\
\text { heuristica } \\
\text { ótimo }\end{array}$ & $\begin{array}{l}\text { Banerjee, } 1965 \text { (veja } \\
\text { Forst. 1984) } \\
\text { Hodgson, } 1977 \quad \text { (veja } \\
\text { Forst, 1984) } \\
\text { Sen e Borah, 1991. } \\
\text { Potts e Van Wasenhover, } \\
1992 \\
\text { Holsenbank e Russel, } \\
1992 \\
\text { Chu, } 1992\end{array}$ & $\begin{array}{l}\text { Tempo de processamento } \\
\text { estocástico. } \\
\text { Tempo de processamento } \\
\text { estocástico. } \\
\text { Interrupçăo e náo } \\
\text { interrupçăo nos produtos. } \\
\begin{array}{l}\text { Datas de liberaçăo } \\
\text { desiguals }\end{array}\end{array}$ \\
\hline
\end{tabular}

Figura 2: Problemas de Um Estágio com Um Processador 


\begin{tabular}{|c|c|c|c|}
\hline Funçäo Objetivo & Método & Reteréncla & Observaçbes" \\
\hline $\bar{T}$ & $\begin{array}{l}\text { houristica } \\
\text { heuristica } \\
\text { heuristica }\end{array}$ & $\begin{array}{l}\text { Wilkinson \& Inwin, } 1971 \\
\text { (veja MacCarthey e Liu, } \\
\text { 1993) } \\
\text { Frey et al. } 1971 \text { (veja } \\
\text { MacCarthey e Liu, 1993) } \\
\text { Panwalkar et al. 1993 }\end{array}$ & \\
\hline$\overline{N_{T}}$ & $\begin{array}{l}\text { otimo } \\
\text { otimo } \\
\text { ótimo }\end{array}$ & $\begin{array}{l}\text { Moore, } 1968 \text { (veja } \\
\text { MacCarthey e Liu, 1993) } \\
\text { Balut, } 1973 \text { (veja Forst, } \\
1984 \text { ) } \\
\text { Adiri et al. } 1991\end{array}$ & $\begin{array}{l}\text { Tempo de processamento } \\
\text { estocastico. } \\
\text { interrupça devido a } \\
\text { quebra de máquina. }\end{array}$ \\
\hline$w_{j} N_{T}$ & otimo & $\begin{array}{l}\text { Potts e Wassenhove, } \\
1988\end{array}$ & \\
\hline$w_{j} T_{j}$ & $\begin{array}{l}\text { otimo } \\
\text { otimo } \\
\text { otimo } \\
\text { heurística } \\
\text { otimo } \\
\end{array}$ & $\begin{array}{l}\text { Lawler, 1964 (veja King e } \\
\text { Spachis, 1980) } \\
\text { Blau, 1973 (veja Forst, } \\
\text { 1984) } \\
\text { Shwimer, 1972 (veja } \\
\text { MacCarthey e Liu, 1993) } \\
\text { Chambers ot al. 1991 } \\
\text { Szwarc e Liu, } 1993\end{array}$ & $\begin{array}{l}\text { Tempos de chegada } \\
\text { diferentes. } \\
\text { Tempo de processamento } \\
\text { estocéstico e "due dates". }\end{array}$ \\
\hline $\bar{F}$ & $\begin{array}{l}\text { otimo } \\
\text { heuristica }\end{array}$ & $\begin{array}{l}\text { Conway, } 1967 \quad \text { (veja } \\
\text { MacCarthey e Liu, 1993) } \\
\text { Liu } \theta \text { MacCarthy, } 1990\end{array}$ & \\
\hline$w_{i} \bar{F}$ & otimo & Mason e Anderson, 1991 & \\
\hline$F V$ & heuristica & Gupta et al. 1990 & \\
\hline VAR,COVAR,BICRIT & $\begin{array}{l}\text { heuristica } \\
\text { simulated annealing }\end{array}$ & Mittenthal et al. 1993 & $\begin{array}{l}\text { Programaç5o ótima de } \\
\text { forma } V \text {. }\end{array}$ \\
\hline$E_{l} / T_{j}$ & $\begin{array}{l}\text { otimo } \\
\text { otimo } \\
\text { otimo }\end{array}$ & $\begin{array}{l}\text { Hall et al. } 1991 \\
\text { Davis e Kanet, } 1993 \\
\text { Szwarc, } 1993\end{array}$ & \\
\hline$E_{j j} / T_{j} \theta S T_{j}$ & heurlstica & Sung e Joo, 1992 & $\begin{array}{l}\text { Produtos com tempos } \\
\text { dinamicos. }\end{array}$ \\
\hline $\begin{array}{l}E_{j} T_{i} \text { e custo de eniraga } \\
\text { de produtos atresedos }\end{array}$ & ótimo & Herrmann e Lee, 1992 & \\
\hline$w_{j} E_{j} / w_{j} T_{j}$ & ótimo & Hall e Posner, 1991 & \\
\hline$T_{\max } \otimes S U P j$ & $\begin{array}{l}\text { heuristica - Tabu } \\
\text { Search }\end{array}$ & $\begin{array}{l}\text { Laguna, et al. 1991, (veja } \\
\text { Barnes e Leguna, 1993) }\end{array}$ & \\
\hline$f_{\max }$ & otimo & Gordon, 1993 & Interrupcáo. \\
\hline$P_{\max }$ & ótimo & De et al. 1993 & $\begin{array}{l}\text { Tempo de processamento } \\
\text { aleatório } \theta \text { "deadline". }\end{array}$ \\
\hline $\begin{array}{l}\text { Min: produçá esperada } \\
\text { a custo de destocamento }\end{array}$ & heurística & $\begin{array}{l}\text { Leachman G Gescon, } \\
1988\end{array}$ & $\begin{array}{l}\text { Closed shop - demanda } \\
\text { estocastica. }\end{array}$ \\
\hline $\begin{array}{l}\text { Min: custo médio de } \\
\text { preperaça, delocamneto } \\
\text { e fore de estoque }\end{array}$ & controle otimo & Gallego, 1990 & $\begin{array}{l}\text { Closed shop - demanda } \\
\text { aleatoria. }\end{array}$ \\
\hline Min: custo de producto & ótimo & Ibrahim e Thomas, 1991 & Closed shop. \\
\hline Min: custo total & otimo & Zheng at al. 1993 & \\
\hline NPV & heurística & Lawrence, 1991 & \\
\hline MWAD & heuristica & Nandkeolyar of al. 1993 & $\begin{array}{l}\text { Chegade estocástica de } \\
\text { produtos }\end{array}$ \\
\hline
\end{tabular}

Figura 2: continuação... 


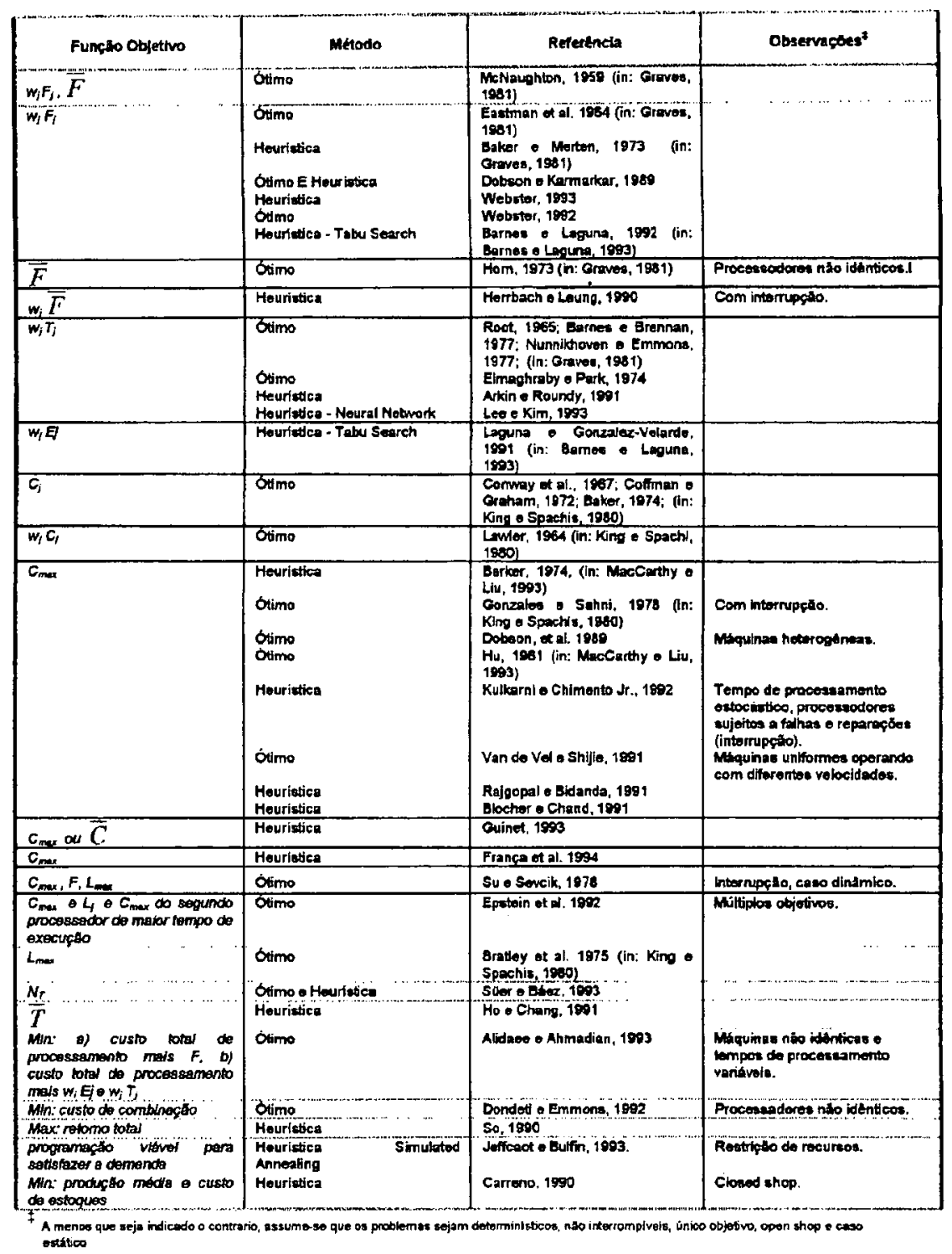

Figura 3: Problema Um Estágio - Processadores Paralelos 


\begin{tabular}{|c|c|c|c|}
\hline Fungto Objetivo & Método & Referencla & Observmbdest \\
\hline$C_{\max }$ & 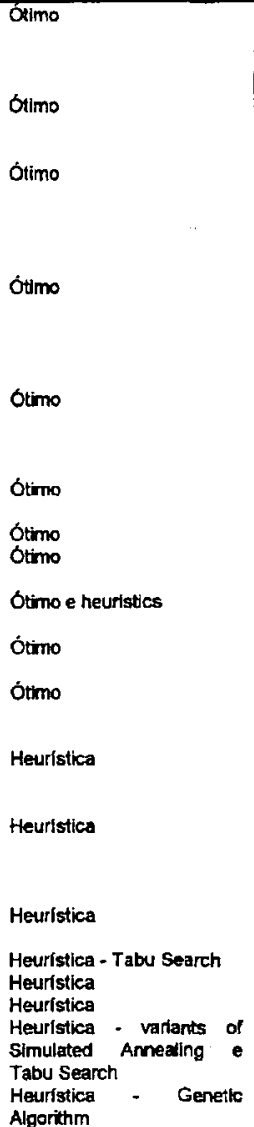 & 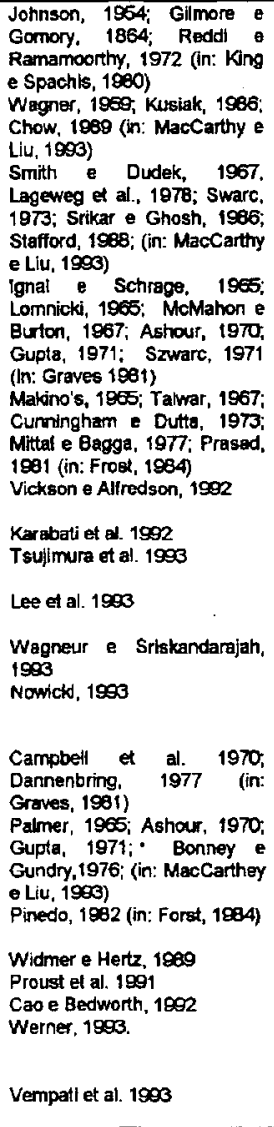 & $\begin{array}{l}\text { Tempo de processamento } \\
\text { estocatico. } \\
\text { Uso de batches de } \\
\text { transfertencia. } \\
\text { Flow shop de permutaçáo. } \\
\text { Tempos de processamento } \\
\text { Inoertas. } \\
\text { Flow shop tipo inha de } \\
\text { montagem. } \\
\text { Flow shop de permutaçá. } \\
\text { Flow shop de permutaçáo. } \\
\text { tempos de processamento } \\
\text { controtaveis. }\end{array}$ \\
\hline $\begin{array}{ll}C_{\text {mas }} & \text { Tempo de } \\
\text { preparacio esperado }\end{array}$ & Otumo & Bagga, 1970 (in: Frost, 1981) & $\begin{array}{l}\text { Tempos de processamento } \\
\text { estocasticos. }\end{array}$ \\
\hline$C_{\operatorname{mix}} \oplus F$ & Stimo & Rajendran, 1992 & Bi-criterios. \\
\hline$\vec{F}$ & Otimo & Cadambi e Sathe, 1993 & \\
\hline$\frac{C_{j}}{T}$ & $\begin{array}{l}\text { Otimo } \\
\text { Otimo }\end{array}$ & $\begin{array}{l}\text { Karabati e Kouvelis, } 1993 \\
\text { KJm, } 1990\end{array}$ & Flow shop de permutaça. \\
\hline $\begin{array}{l}\text { Achar nequeimentos de } \\
\text { demanda ao minimo custot }\end{array}$ & $\begin{array}{l}\text { Ótimo } \\
\text { Heurfstica }\end{array}$ & $\begin{array}{l}\text { Zangwill, } 1969 \text {, Love, } 1972 \\
\text { (in: Graves, } 1981 \text { ) } \\
\text { Lambrecht o Vander Eecken, } \\
1978 \text { (In: Graves, } 1981 \text { ) }\end{array}$ & $\begin{array}{l}\text { Closed shop. } \\
\text { Demanda varktvel. }\end{array}$ \\
\hline Min: cutso total esperedo & & Forst, 1983 & $\begin{array}{l}\text { Tempos de processamento } \\
\text { estocastl cos. }\end{array}$ \\
\hline
\end{tabular}

\section{Figura 4: Problemas Múltiplos Estágios - Flow Shop}




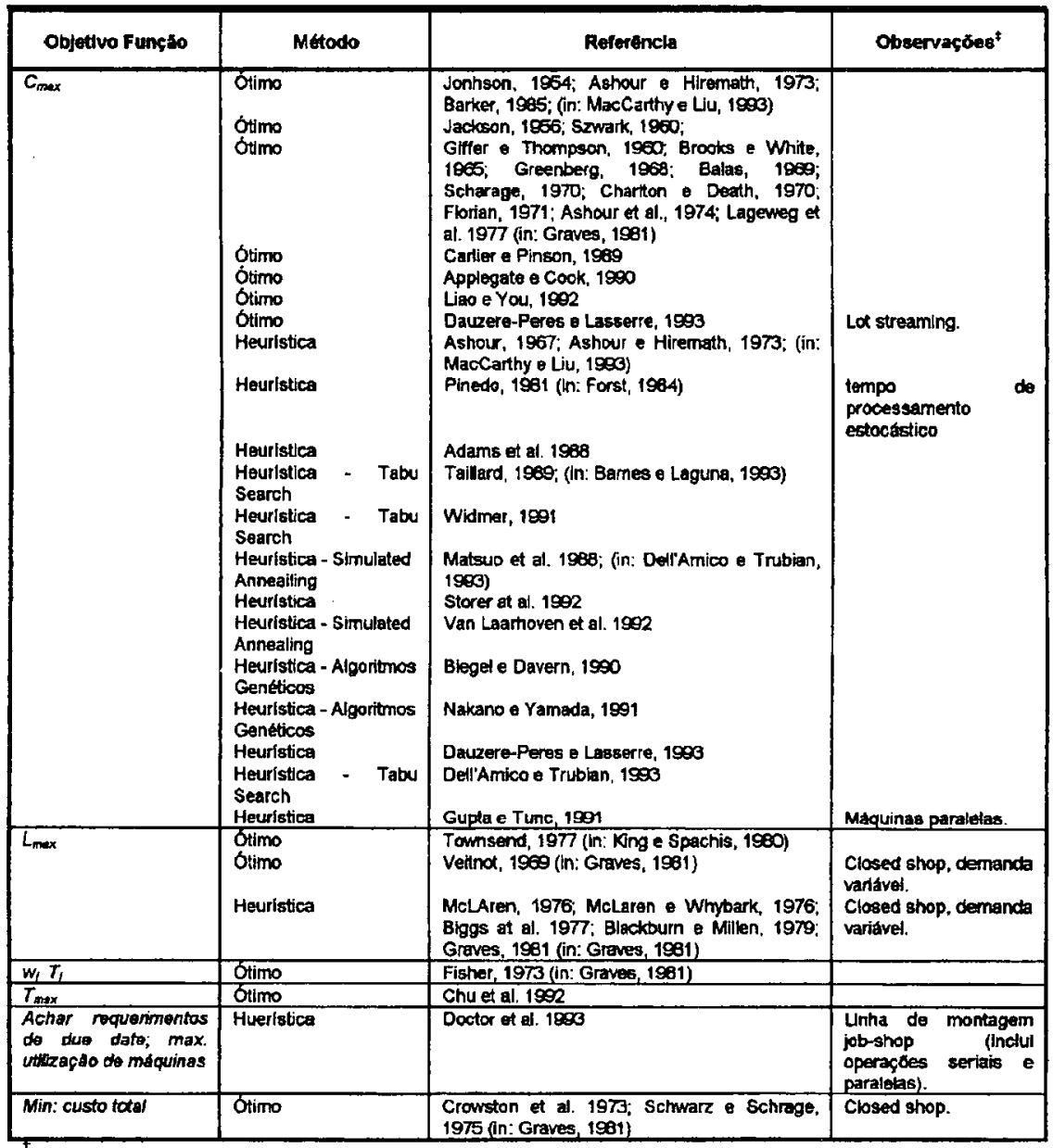

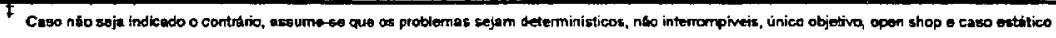

\section{Figura 5: Problemas Múltiplos Estágios - Job Shop}




\section{Conclusão}

O campo da programação da produção é muito dinâmico e reconhecidamente, começou no início dos anos $50 \mathrm{com}$ o trabalho pioneiro de Johnson (1954) e desde então a literatura tem crescido explosivamente. $O$ trabalho propõe um esquema para a classificação dos problemas de programação baseado nas características gerais dos problemas e as hipóteses usuais feitas sobre os produtos, máquinas e tempos de processamento. Este esquema, que pode ser considerada como uma macro-divisãò dos problemas de programação da produção, objetiva estabelecer o sentido, direção e perspectiva de pesquisas conduzidas na área.

\section{Notas}

1. Regras de dispatching e sequenciamento fornecem uma solução para o problema de programação empregando regras locais para a seleção de tarefas (Matsuura et al., 1993, Chryssolouris et al., 1992), enquanto que nos sistemas especialistas, as regras aplicadas pelo homem para atribuir as tarefas aos recursos, são codificada numa base de regras a qual é invocada toda vez que uma decisão de atribuição necessita ser feita (Chryssolouris et al., 1992).

2. Cada iteração começa de um estado de solução (que representa o conjunto de soluções). É daqui que as candidatas devem ser determinadas e avaliadas de forma que o estado de solução da próxima iteração (ou próximas iterações) possa ser escolhido. $\mathrm{O}$ conjunto de candidatas é denominado a vizinhança do estado (Müller-Merbach, 1981).

3. Máquina idêntica é um equipamento alternativo num dado estágio que permite executar certa operação com tempo de processamento idêntico e, ainda, características e demanda de recursos iguais. 


\section{Referências Bibliográficas}

ADAMS, J., BALAS, E. e ZAWACK, D. The Shifting Bottleneck Procedure For Job Shop Scheduling. Management Science. v.34, n.3, p. 391-401. 1988.

ADIRI, I., FROSTIG, E. e RINNOOY KAN, A.H.G. Scheduling On A Single Machine With A Single Breakdown To Minimize Stochastically The Number Of Tardy Jobs. Naval Research Logistics, v. 38, p. 261-271. 1991.

ALIDAEE, B. A Heuristic Solution Procedure To Minimize Makespan On A Single Machine With Non-Linear Cost Functions. Journal of Operational Research Society, v. 41, n.11, p. 1065-1068. 1990.

ALIDAEE, B. e AHMADIAN, A. Two Parallel Machine Sequencing Problems Involving Controllable Job Processing Times. European Journal of Operational Research, v. 70, p. 335-341. 1993.

APPLEGATE, D. e COOK, W. A Computational Study Of The Job-Shop Scheduling Problem. ORSA Journal of Computation. v. 3, p. 149-156. 1990.

ARKIN, E.M. e ROUNDY, R.O. Weighted-Tardiness Scheduling On Parallel Machines With Proportional Weights. Operations Research, v. 39, n. 1, p. 64 - 81.1991.

BAKER, K. e SCUDDER, G.D. Sequencing With Earliness And Tardiness Penalties: A Review. Operations Research. v. 38, p. 22-36. 1990.
BAKSHI, M.S. e ARORA, S.R. The Sequencing Problem. Management Science. v. 16, p. B247-B263. 1969.

BARNES, J.W. e LAGUNA. M. A Tabu Search Experience In Production Scheduling. An, s of Operations Research. v. 41, p. 141-156. 1993.

BELLMAN, R., ESOGBUE, A.O. e NABESHIMA, I. Mathematical Aspects of Scheduling And Applications. Pergamon Press. 1982.

BIEGEL, J.E. e DAVERN, J.J. Genetic Algorithms And The Job Shop Scheduling. Computers and Industrial Engineering. v. 19, n. 1 e 4, p. 81-91. 1990.

BLOCHER, J.D. e CHAND, S. Scheduling Of Parallel Processors: A Posterior Bound On LPT Sequencing And A Two-Step Algorithm. Naval Research Logistics. v. 38, p. 273-287. 1991.

BUXEY, G. Production Scheduling: Practice And Theory. European Journal of Operational Research. v. 39, p. 17-31. 1989.

CADAMBI, B.V. e SATHE, Y.S. Two-Machine Flowshop Scheduling To Minimize Mean Flow Time. Opsearch. v. 30, n. 1, p. 34-41. 1993.

CAO, J. e BEDWORTH, D.D. Flow Shop Scheduling In Serial Multi-Product Processes With Transfer And Set-Up 
Times. International Journal of Production Research, v. 30, n. 8, p. 1819-1830. 1992.

CARLIER, J. e PINSON, E. An Algorithm For Solving The Job-Shop Problem. Management Science. v. 35, n. 2, p. 164-176. 1989.

CARRENO, J.J. Economic Lot Scheduling For Multiple Products On Parallel Identical Processors. Management Science. v. 36, n. 3, p. 348-358. 1990.

CHAMBERS, R.J., CARRAWAY, R.L., LOWE, T.J e MORIN, T.L. Dominance And Decomposition Heuristics For Single Machine Scheduling. Operations Research. v. 39, n. 4, p. 639 648. 1991.

CHANDRU, V., LEE, C.-Y. e UZSOY, R. Minimizing Total Completion Time On Batch Processing Machines. International Journal of Production Research. v. 31, n. 9, p. 2097-2121. 1993.

CHRYSSOLOURIS, G., DICKE, K. e LEE, M. On The Resource Allocation Problem. International Journal of Production Research. v.31, n.12, p. 2773-2795. 1992.

CHENG, C. e BULFIN R.L. Complexity of Single Machine, Multi-Criteria Scheduling Problems. European Journal of Operational Research. v. 70, p. 115-125. 1993.

CHU, C. A Branch-And-Bound Algorithm To Minimize Total Flow Time With Unequal Release Dates. Naval
Research Logistics. v. 39, p. 859-875. 1992a.

CHU, C. A Branch-And-Bound Algorithm To Minimize Total Tardiness With Different Release Dates. Naval Research Logistics. v. 39, p. 265-283. 1992.

CHU, C., PORTMANN, M.C. e PROTH, J.M. A Splitting-Up Approach To Simplify Job-Shop Scheduling Problems. International Journal of Production Research. v. 30, n. 4, p. 859-870. 1992.

COFFMAN, JR. E.G. Introduction To Deterministic Scheduling Theory. In: Computer And Job-Shop Scheduling Theory. Coffman, Jr.E.G. John Wiley \& Sons. 1976.

DAUZERE-PERES, S. The One-Machine Sequencing Problem With Dependent Jobs. Computer and Industrial Engineering., v. 25, n. 1/44, p. 235-238. 1993.

DAUZERE-PERES, S. e LASSERRE, J-B. An Iterative Procedure For Lot Streaming In Job-Shop Scheduling. Computers and Industrial Engineering. v. 25, n. 1/4, p. 231-234. 1993a.

DAUZERE-PERES, S. e LASSERRE, J-B. A Modified Shifting Bottleneck Procedure For Job-Shop Scheduling. International Journal of Production Research. v. 31, n. 4, p. 923-932. 1993.

DAVIS, J.S. e KANET, J.J. Single=Machine Scheduling With Early And Tardy Completion Costs. Naval Research Logistics. v. 40, p. 85-101. 1993. 
DAY, J.E e HOTTENSTEIN, M.P. Review Of Sequencing Research. Naval Research Logistics Quarterly. v. 17, p. 11-40. 1970,

DE, P., GHOSH, J.B. e WELLS, C.E. On The Minimization Of Time Variance With Bicriteria Extension. Operations Research. v. 40, n. 6, p. 1148-1155. 1992.

DE, P., GHOSH, J.B. e WELLS, C.E. Job Selection And Sequencing On A Single Machine In A Random Environment. European Journal of Operational Research. v. 70, p. 425-431. 1993.

DELL'AMICO, M. e TRUBIAN, $M$. Applying Tabu Search To The Job-Shop Scheduling Problem. Annals of Operations Research. v. 41, p. 231-252. 1993.

DOBSON, G. e KARMARKAR, U.S. Simultaneous Resource Scheduling To Minimize Weighted Flow Times. Operations Research. v. 37, n. 4, p. 592-600. 1989.

DOBSON, G., KARMARKAR, U.S. e RUMMEL, J.L. Batching To Minimize Flow Times On Parallel Heterogeneous Machines. Management Science. v. 35, n. 5, p. 607-613. 1989.

DOCTOR, S.R., CAVALIER, T.M. e EGBE:U, P.J. Scheduling For Machining And Assembly In A Job-Shop Environment. International Journal of Production Research. v. 31, n. 6, p. 1275-1297. 1993.

DONDETI, V.T. e EMMONS, H. Fixed Job Scheduling With Two Types Of
Processors. Operations Research. v. 40, supp.1, p. S76. 1992.

ELMAGHRABY, S.E. The Machine Sequencing Problem - Review And Extensions. Naval Research Logistics Quarterly. v. 15, p. 205-232. 1968.

EPSTEIN, S., WILAMOWSKY, Y. e DICKMAN, B. Deterministic Multiprocessor Scheduling With Multiple Objectives. Computers Operations Research. v. 19, n. 8, p. 743-749. 1992.

FORST, F.G. Minimizing Total Expected Costs In The Two-Machine, Stochastic Flow Shop. Operations Research Letters. v. 2, p. 58-61. 1983.

FORST, F.G. A Review Of The Static, Stochastic Job Sequencing Literature. Opsearch. v. 21, p. 127-144. 1984.

FRANÇA, P.M., GENDREAU, M., LAPORTE, G. e MÜLLER, F.M. A Composite Heuristic For The Identical Parallel Machine Scheduling Problem With Minimum Makespan Objective. Computer Operations Research. v. 21, n. 2, p. 205-210. 1994.

GALLEGO, G. Scheduling The Production Of Several Items With Random Demands In A Single Facility. Management Science. v. 36, n. 12, p. 1579-1592. 1990.

GAREY, M.R., JOHNSON, D.S. e SETHI, R. The Complexity Of Flowshop And Jobshop Scheduling, Mathematics of Operations Research. v. 1, p. 117-129. 1976. 
GLAZEBROOK, K.D. Single-Machine Scheduling Of Stochastic Jobs Subject To Deterioration Or Delay. Naval Research Logistics. v. 39, p. 613-633. 1992.

GODIN, V.B. Interactive Scheduling: Historical Survey And State Of The Art. AIIE Trans. v. 10, p. 331-337. 1978.

GOYAL, S.K. e SRISKANDARAJAH, C. No-Wait Shop Scheduling: Computational Complexity And Approximate Algorithms. Opsearch. v. 25, p. 220-244. 1988.

GORDON, V.S. A Note On Optimal Assignment Of Slack Due-Dates In Single-Machine Scheduling. European Journal of Operational Research. v. 70, p. 311-315. 1993.

GRAHAM, R.L., LAWLER, E.L., LENSTRA, J.K. e RINNOOY KAN, A.H.G. Optimization And Approximation In Deterministic Sequencing And Scheduling: A Survey. Annals of Discrete Mathematics. v. 5, p. 287-326. 1979.

GRAVES, S.G. A Review of Production Scheduling. Operations Research. v. 29, p. 646-675. 1981.

GUINET, A. Scheduling Sequence-Dependent Jobs On Identical Parallel Machines To Minimize Completion Time Criteria. International Journal of Production Research. v. 31, n. 7, p. 1579-1594. 1993.

GUPTA, J.N.D. e TUNC, E.A. Scheduling For A Two-Stage Hybrid
Flowshop With Parallel Machines At The Second Stage. International Journal of Production Research. v. 29, n. 7, 1489-1502. 1991.

GUPTA, M.C., GUPTA, Y.P. e BECTOR, C.R. Minimizing The Flow-Time Variance In Single-Machine Systems. Journal of Operational Research Society. v. 41, n. 8, p. 767-779. 1990.

GUPTA, M.C., GUPTA, Y.P. e KUMAR, A. Minimizing Flow Time Variance In A Single Machine System Using Genetic Algorithms. European Journal of Operational Research. v. 70, p. 289-303. 1993.

HALL, N.G. e POSNER, M.E. Earliness-Tardiness Scheduling Problems, I: Weighted Deviation Of Completion Times About A Common Due Date. Operations Research. v. 39, n. 5, 836-846. 1991.

HALL, N.G., KUBIAK, W e SETHI, S.P. Earliness-Tardiness Scheduling Problems, II: Deviation Of Completion Times About A Restrictive Common Due Date. Operations Research. v. 39, n. 5, p. 836-847. 1991.

HENDRY, L.C. e KINGSMAN, B.G. Production Planning Systems And Their Applicability To Make-To-Order Companies. European Journal of Operational Research. v. 40, p.1-15. 1989.

HERRBACH, L.A. e LEUNG, J.Y.-T. Preemptive Scheduling Of Equal Length Jobs On Two Machine To Minimize Mean 
Flow Time. Operations Research. v. 38, n. 3), 487-494. 1990.

HERRMANN, J.W. e LEE, C. On Scheduling To Minimize Earliness-Tardiness And Batch Delivery Costs With A Common Due Date. European Journal of Operational Research. v. 70, p. 272-288. 1992.

HO, J.C., e CHANG, Y.-L. Heuristic For Minimizing Mean Tardiness For $M$ Parallel Machines. Naval Research Logistics. v. 38, p. 367-381. 1991.

HOLSENBACK, J.E. e RUSELL, R.M. A Heuristic Algorithm For The Sequencing On One Machine To Minimize Total Tardiness. Journal of Operational Research Society. v. 43, n. 1, p. 53-62. 1991.

IBARAKI, T. Combinatorial Optimization Problems And Their Complexity, In: Enumerative Approaches To Combinatorial Optimization - Part I. Annals of Operations Research, v. 10. 1987.

IBRAHIM, A. e THOMAS, L.C. A Two-Product, Single-Machine, Storage-Constrained Production Problem. Journal of Operational Research Society. v. 42, n. 9, p. 785-791. 1991.

JEFFCOAT, D.E. e BULFIN, R.L. Simulated Annealing For Resource-Constrained Scheduling. European Journal of Operational Research. v. 70. p. 43-51. 1993.
KAMOUN, H. e SRISKANDARAJAH, C. The Complexity Of Scheduling Jobs In Repetitive Manufacturing Systems. European Journal of Operational Research. v. 70, p. 350-364. 1993.

KARABATI, S., KOUVELIS, P. e KIRAN, A.S. Games, Critical Paths And Assignment Problems In Permutation Flow Shops And Cyclic Scheduling Flow Line Environments. Journal of the Operational Research Society. v. 43, n. 3, p. 241-258. 1992.

KARABATI, S., KOUVELIS, P. Completion Times Performance Criterion. Naval Research Logistics. v. 40, p. 843-862. 1993.

KIM, Y.D. A New Branch And Bound Algorithm For Minimizing Mean Tardiness In Two-Machine Flowshops. Computers Operations Research. v. 20, n. 4, p. 391-401. 1993.

KING, J.R. e SPACHIS, A.S. Scheduling: Bibliography \& Review. International Journal of Physical Distribution and Materials Management. v. 10, n. 3, p. 105-132. 1980.

KIRAN, A.S. E UNAL, A.T. A Single-Machine Problem With Multiple Criteria. Naval Research Logistics. v. 38, p. 721-727. 1991.

KULKARNI, V.G. e CHIMENTO Jr., P.F. Optimal Scheduling Of Exponential Task With In-Tree Precedence Constraints On Two Parallel Processors Subject To Failure And Repair. Operations Research. v. 40, supp. n. 2, p. S263. 1992. 
LAWRENCE, S.R. Scheduling A Single Machine To Maximize Net Present Value. International Journal of Production Research. v. 29, n. 6, p. 1141-1160. 1991.

LEACHMAN, R.C. e GASCON, A. A Heuristic Scheduling Policy For Multi-Item, Single-Machine Production Systems With Time-Varying, Stochastic Demands. Management Science. v. 34, n. 3, p. 377-390. 1988.

LEE, I. A Worst-Case Performance Of The Shortest-Processing-Time Heuristic For Single Machine Scheduling. Journal of The Operational Research Society. v. 42, n. 10, p. 895-901. 1991.

LEE,Y.H. e KIM, S. Neural Network Application For Scheduling Jobs On Parallel Machines. Computer and Industrial Engineering. v. 25, n.1/4, p. 227-230. 1993.

LEE, C.Y., CHENG, T.C.E. e LIN, B.M.T. Minimizing The Makespan In The 3-Machine Assembly-Type Flowshop Scheduling Problem. Management Science. v. 39, n. 5, p. 616-625. 1993.

LEON, V.J. e WU S.D. On Scheduling With Ready-Times, Due-Dates And Vacations. Naval Research Logistics. v. 39, p. 53-65. 1992.

LENSTRA, J.K. Sequencing By Enumerative Methods. Mathematical Centre Tracts 69, Amsterdam. 1977.

LENSTRA, J.K. e RINNOOY KAN, A.H.G. Complexity Of Scheduling Under Precedence Constraints, Operations Research. v. 26, n. 1, p. 22-35. 1978
LENSTRA, J.K., RINNOOY KAN, A.H.G., e BRUCKER, P. Complexity of Machine Scheduling Problems, Annals of Discrete Mathematics. v. 1, p. 343-362. 1977

LIAO, C.J. e YOU, C.T. An Improved Formulation For The Job-Shop Scheduling Problem. Journal of the Operational Research. v. 43, n. 11, p. 1047-1054. 1992.

LIU, J. e MacCARTHY, B.L. Effective Heuristics For The Single Machine Sequencing Problem With Ready Times. International Journal of Production Research. v. 29, n. 8, p. 1521-1533. 1990.

MACCARTHY B.L e LIU J. Addressing The Gap In Scheduling Research: A Review Of Optimization And Heuristic Methods In Production Scheduling. International Journal of Production Research. v. 31, n. 1, p. 59-79. 1993.

MASON, A.J. e ANDERSON, E.J. Minimizing Flow Time On A Single Machine With Job Classes Setup Times. Naval Research Logistics. v. 38, p. 333-350. 1991.

MATSSURA, H., TSUBONE, H. e KANEZASHI, M. Sequencing, Dispatching, And Switching In A Dynamic Manufacturing Environment. International Journal of Production Research. v. 31, n. 7 , p. 1671-1688. 1993

MÜLLER-MERBACH, H. Heuristic And Their Design: A Survey. European Journal of Operational Research. v. 8, p. 1-23. 1981. 
MITTENTHAL, J., RAGHAVACHARI, M. e RANAS, A. A Hybrid Simulated Annealing Approach For Single Machine Scheduling Problems With Non-Regular Penalty Functions. Computer Operational Research. v. 20, n. 2, p. 103-111. 1993.

MONMA C.L. e POTTS C.N. On The Complexity Of Scheduling Batch Setup Times. Operations Research. v. 37, p. 798-804. 1989.

NAKANO, R. e YAMADA, T. Conventional Genetic Algorithm For Job Shop Problems. Proc. 4th. Int. Conf. on Genetic Algorithms, San Diego, CA. p. 474-479. 1991.

NANDKEOLYAR, U., AHMED, M.U. e SUNDARARAGHAVEN, P.S. Dynamic Single-Machine-Weighted Absolute Deviation Problem: Predictive Heuristics And Evaluation. International Journal of Production Research. v. 31, n. 6, p. 1453-1466. 1993.

NOWICKI, E. An Approximation Algorithm For The $M$-Machine Permutation Flow Shop Scheduling With Controllable Processing Times. European Journal of Operational Research. v. 70, p. 342-349. 1993.

PANWALKER, S.S. e ISKANDER, W. A Survey Of Scheduling Rules. Operations Research. v. 25, p. 45-61. 1977.

PANWALKER, S.S., SMITH, M.L. e KOULAMAS, C.P. A Heuristic For Single Machine Tardiness Problem. European Journal of Operational Research. v. 70, p. 304-310. 1993.
POTTS, C.N. e VAN WASSENHOVE, L.N.. Algorithms For Scheduling A Single Machine For Minimizing The Weighted Number Of Late Jobs. Management Science. v. 34, n. 7, p. 843-858. 1988

POTTS, C.N. e VAN WASSENHOVE, L.N. Single Machine Scheduling To Minimize Total Late Work. Operations Research. v. 40, n. 3, p. 586-595. 1992.

PROUST, C., GUPTA, J.N.D. e DESCHAMPS, V. Flowshop Scheduling With Set-Up, Processing And Removal Times Separated. International Journal of Production Research. v. 29, n. 3, p. 479-493. 1991.

RAJENDRAN, C. Two-Stage Flowshop Scheduling Problem With Bicriteria. Journal of the Operational Research Society. v. 43, n. 9, p. 871-884. 1992.

RAJGOPAL, J. e BIDANDA, B. On Scheduling Parallel Machines With Two Setup Classes. International Journal of Production Research. v. 29, n. 12, p. 2243-2458. 1991.

RINNNOY KAN, H.H.G. Machine Scheduling Problems: Classification, ComplexityAnd Computations. The Hague: Nijhoff. 1976.

RODAMMER, F.A. e WHITE, Jr. K.P. A Recent Survey Of Production Scheduling. IEEE Transaction on Systems, Man and Cybernetics. v. 18, n. 6, p. 841-851. 1988. 
SEN, T. e BORAH, B.N. On The Single-Machine Scheduling Problem With Tardiness Penalties. Journal of Operations Research Society. v. 42, n. 8, p. 695-702. 1991.

SO, K.C. Some Heuristics For Scheduling Jobs On Parallel Machines With Setups. Management Science. v. 36, n. 4, p. 467 - 475. 1990.

STORER, H.R., WU, S,W. e VACCARI, R. New Search Spaces For Sequencing Problems With Application To Job Shop Scheduling. Management Science. v. 38, n. 10, p. 1495-1509. 1992.

\section{SU, Z.S. e SEVCIK, K.C. A} Combinatorial Approach To Dynamic Scheduling Problems. Operations Research. v. 26, n. 5, p. 836-844. 1978.

SÜER, G.A. e BÁEZ. Minimizing The Number Of Tardy Jobs In Identical Machine Scheduling. Computer and Industrial Engineering. v. 25, n. 1/4, p. 243-246. 1993.

SUNG. C.S. e JOO, U.G. A Single Machine Scheduling Problem With Earliness/Tardiness And Starting Time Penalties Under A Common Due Date. Computers and Operational Research. v. 19, n. 8, p. 757-766. 1992.

SZWARC, W. Adjacent Orderings In Single-Machine Scheduling With Earliness And Tardiness Penalties. Naval Research Logistics. v. 40, p. 229-243. 1993.

SZWARC, W., POSNER, M.E. e LIU, J.L. The Single Machine Problem With A
Quadratic Cost Function Of Completion Time. Management Science. v. 34, n. 12, p. 1480-1488. 1988.

SZWARC, W. e LIU, J.J. Weighted Tardiness Single Machine Scheduling With Proportional Weights. Management Science. v. 39, n. 5, p. 626-632. 1993.

TSUJIMURA, Y., PARK, S.H., CHANG, I.S. e GEN, M. An Effective Method For Solving Flow Shop Scheduling Problems With Fuzzy Processing Times. Computers and Industrial Engineering. v. 25, n. 1/4, p. 239-242. 1993.

ULLMAN, J.D., NP-Complete Scheduling Problems. Journal of Computing System Science. v. 10, p. 384-393. 1975

VAN DE VEL, H. e SHIJIE, S. An Application of The Bin-Packing Technique To Job Scheduling On Uniform Processors. Journal of The Operational Research Society. v. 42, n. 2, p. 169-172. 1991.

VAN LAARHOVEN, P.J.M., AARTS, E.H.L. e LENSTRA, J.K. Job Shop Scheduling By Simulated Annealing. Operations Research. v. 40, n. 1, p. 113-125. 1992.

VEMPATI, V.S., CHEN, C.-L. e BULLINGTON, S.F. An Effective Heuristic For Flow Shop Problems With Total Flow Time As Criterion. Computers and Industrial Engineering. v. 5, n. 1/4, p. 219-222. 1993. 
VICKSON, R.G. e ALFREDSSON, B.E. Two And Three Machine Flow Shop Scheduling Problems With Equal Sized Transfer Batches. International Journal of Production Research, v. 30, n. 7, p. 1551-1574. 1992.

WAGNEUR, E. e SRISKANDARAJAH, C. The Two-Machine Permutation Flow Shop With State-Dependent Processing Times. Naval Research Logistics. v. 40, p. 697-717. 1993.

WEBSTER, S. New Bounds For The Identical Parallel Processor Weighted Flow Time Problem. Management Science. v. 38, n. 1, p. 124-136. 1992.

WEBSTER, S.T. A Priority Rule For Minimizing Weighted Flow Time In A Class Of Parallel Machine Scheduling Problems. European Journal of Operational Research. v. 70, p. 327-334. 1993.

WERNER, F. On The Heuristic Solution Of The Permutation Flow Shop Problem By Path Algorithms. Computers Operations Research. v. 20, n. 7, p. 707-722. 1993.

WIDMER, M. Job Shop Scheduling With Tooling Constraints: A Tabu Search Approach. Journal of the Operational Research Society. v. 42, n. 1, p. 75-82. 1991.

WIDMER, M. e HERTZ, A. A New Method For The Flow Sequencing Problem. European Journal of Operational Research. v. 41, p. 186-193. 1989.
WU, S.D., STORES, R.H. and CHANG, P. One-Machine Rescheduling Heuristic With Efficiency And Stability As Criteria. Computer Operational Research. v. 20, n. 1, p. 1-14. 1993.

ZHENG, W.-X., NAGASAWA, H. e NISHIYAMA, N. Single-Machine Scheduling For Minimizing Total Cost With Identical, Asymmetrical Earliness And Tardiness Penalties. International Journal of Production Research. v. 31, n. 7, p. 1611-1620. 1993. 